\title{
The tough body at the epicentre of amyotrophic lateral sclerosis
}

\section{The corpus callosum is a signpost to the interhemispheric highways underpinning the widespread cerebral pathology that typifies the syndrome of ALS}

\author{
Martin R Turner, Ricarda A L Menke
}

The brain has become axiomatic to understanding pathogenesis in amyotrophic lateral sclerosis (ALS), not least given the clinical, molecular and genetic overlap with frontotemporal dementia. ${ }^{1}$ ALS, although defined in part by its lower motor neuron degeneration, now appears misfiled alongside the neuromuscular disorders. The corpus callosum (CC, latin translation: tough body) is a pervasive cerebral integrator, and a consistent part of the in vivo cerebral white matter MRI signature of $\mathrm{ALS},{ }^{2}$ noted in post mortem studies many decades prior to diffusion tensor imaging (DTI). ${ }^{3}{ }^{4}$ The JNNP paper by Zhang et $a l^{5}$ uses advanced MRI analysis to explore symmetrical interhemispheric functional connectivity in ALS in relation to the regional anatomy of the CC.

Loss of interhemispheric connectivity in ALS was initially demonstrated in patients using transcranial magnetic stimulation. ${ }^{6}$ The identification of regionally coherent MRI blood oxygenation level-dependent signals in a task-free state (resting-state functional MRI) has been a major driver in understanding brain function as a series of network-based processes. This technique first identified interhemispheric impairment between bilateral subregions of the primary motor cortex in ALS. ${ }^{7} \mathrm{MR}$ tractography can be used to parcellate the CC and create a cross-sectional 'key' to all structurally connected regions of the brain. ${ }^{8}$ Zhang et al combined aspects of both approaches, creating a novel voxel mirrored homotopic map to study the symmetrical functional connectivity of regions in relation to the position of their interhemispheric fibres within five regions along the midline sagittal plane of the CC. The central motor cortical interhemispheric fibres were the most

Nuffield Department of Clinical Neurosciences, Oxford University, Oxford, UK

Correspondence to Professor Martin R Turner, Nuffield Department of Clinical Neurosciences, Oxford University, West Wing Level 6, John Radcliffe Hospital, Oxford OX3 9DU, UK; martin.turner@nden.ox.ac.uk consistently involved in combined functional and structural analyses, the latter in keeping with a previous study that focused on this CC regionality, ${ }^{9}$ and the area where the inflammatory infiltrates appear concentrated. ${ }^{10}$

Subcortical structures, including the putamen, also showed reduced symmetrical functional connectivity and between the superior temporal gyri, the latter aligning with the recognition of consistent verbal fluency impairments in ALS. Cognition was not explored in this study (those with significant cognitive impairment were excluded), though more anterior regions of the CC have been previously linked to measures of frontal lobe dysfunction in ALS. ${ }^{11}$ Nonetheless, symmetrical functional connectivity changes were noted by Zhang et al in multiple bihemispheric regions connected through anterior and posterior parts of the CC, as one would predict with the concept of ALS as a widespread cerebral network disorder, despite its motor-predominant phenotype.

The authors point out that the brain is not symmetrical, which may distort phenotype correlations in relation to voxel mirrored homotopic connectivity. The onset of symptoms in ALS is typically focal and strikingly asymmetric for most of the two-thirds of patients where it begins in a limb. Limb dominance influences laterality of first weakness in upper limb-onset ALS, ${ }^{12}$ and there is a higher probability of contralateral versus ispilateral sequential limb involvement. ${ }^{13}{ }^{14}$ The DTI white matter signature is consistently symmetrical however. ${ }^{15}$ This may reflect group-level analysis in what is a clinically heterogeneous syndrome. Furthermore, there is an average diagnostic delay of $\sim 1$ year in ALS, so that bihemispheric pathology (if not symptoms as well) may be established by the time individuals reach the MRI scanner. With evolving ideas about transsynaptic transmission of pathology in ALS, a natural question is whether the CC facilitates interhemispheric spread. A potentially disease-modifying effect of callosotomy in the presymptomatic period is then a compelling concept, and potentially testable in appropriate non-human models.

In the context of assessing the effects of other therapeutic interventions, the CC, uniquely spanning the breadth of ALS cerebral pathology, warrants further assessment as both a phenotype stratifying and potentially pharmacodynamic biomarker.

Contributors MRT drafted the commentary. RALM edited the commentary.

Funding Medical Research Council. Grant number MR/K01014X/1.

Competing interests None declared.

Provenance and peer review Commissioned; internally peer reviewed.

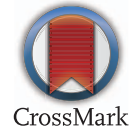

To cite Turner MR, Menke RAL. J Neurol Neurosurg Psychiatry 2017;88:369-370.

Received 12 November 2016

Accepted 20 November 2016

Published Online First 13 December 2016

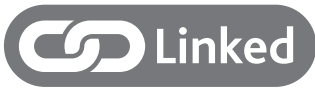

- http://dx.doi.org/10.1136/jnnp-2016-314567

J Neurol Neurosurg Psychiatry 2017;88:369-370. doi:10.1136/jnnp-2016-315247

\section{REFERENCES}

1 Burrell JR, Halliday GM, Kril JJ, et al. The frontotemporal dementia-motor neuron disease continuum. Lancet 2016;388:919-31.

2 Filippini N, Douaud G, Mackay CE, et al. Corpus callosum involvement is a consistent feature of amyotrophic lateral sclerosis. Neurology 2010;75:1645-52.

3 Smith MC. Nerve fibre degeneration in the brain in amyotrophic lateral sclerosis. I Neurol Neurosurg Psychiatry 1960;23:269-82.

4 Turner MR. Nerve fibre degeneration in the brain in amyotrophic lateral sclerosis. I Neurol Neurosurg Psychiatry 2012;83:382.

5 Zhang, et al. Aberrant interhemispheric homotopic functional and structural connectivity in amyotrophic lateral sclerosis. J Neurol Neurosurg Psychiatry 2017:88:374-80.

6 Wittstock M, Wolters A, Benecke R. Transcallosal inhibition in amyotrophic lateral sclerosis. Clin Neurophysiol 2007;118:301-7.

7 Jelsone-Swain LM, Fling BW, Seidler RD, et al. Reduced interhemispheric functional connectivity in the motor cortex during rest in limb-onset amyotrophic lateral sclerosis. Front Syst Neurosci 2010;4:158.

8 Chao YP, Cho KH, Yeh CH, et al. Probabilistic topography of human corpus callosum using cytoarchitectural parcellation and high angular resolution diffusion imaging tractography. Hum Brain Mapp 2009;30:3172-87.

9 Muller HP, Unrath A, Huppertz HJ, et al. Neuroanatomical patterns of cerebral white matter 


\section{Editorial commentary}

involvement in different motor neuron diseases as studied by diffusion tensor imaging analysis. Amyotroph Lateral Scler 2012;13:254-64.

10 Sugiyama M, Takao M, Hatsuta $\mathrm{H}$, et al. Increased number of astrocytes and macrophages/microglial cells in the corpus callosum in amyotrophic latera sclerosis. Neuropathology 2013;33:591-9.

11 Kolind S, Sharma R, Knight $S$, et al. Myelin imaging in amyotrophic and primary lateral sclerosis.
Amyotroph Lateral Scler Frontotemporal Degener 2013;14:562-73.

12 Turner MR, Wicks P, Brownstein CA, et al. Concordance between site of onset and limb dominance in amyotrophic lateral sclerosis. J Neurol Neurosurg Psychiatry 2010;82:853-4.

13 Turner MR, Brockington A, Scaber J, et al. Pattern of spread and prognosis in lower limb-onset ALS. Amyotroph Lateral Scler 2010;11:369-73.
14 Devine MS, Kiernan MC, Heggie S, et al. Study of motor asymmetry in ALS indicates an effect of limb dominance on onset and spread of weakness, and an important role for upper motor neurons. Amyotroph Lateral Scler Frontotemporal Degener 2014;15:481-7.

15 Menke RA, Korner S, Filippini N, et al. Widespread grey matter pathology dominates the longitudinal cerebral MRI and clinical landscape of amyotrophic lateral sclerosis. Brain 2014;137(Pt 9):2546-55. 